\title{
Ferroelectricity and lattice distortion associated with spin orderings in a multiferroic delafossite $\mathrm{AgFeO}_{2}$
}

\author{
Noriki Terada ${ }^{1,2,3, a}$, Dmitry D. Khalyavin ${ }^{2}$, Pascal Manuel ${ }^{2}$, Yoshihiro Tsujimoto ${ }^{1}$, Kevin Knight ${ }^{2}$, Paolo G. Radaelli ${ }^{3}$, \\ Hiroyuki S. Suzuki ${ }^{1}$, and Hideaki Kitazawa ${ }^{1}$ \\ 1 National Institute for Materials Science, Sengen 1-2-1, Tsukuba, Ibaraki 305-0047, Japan \\ 2 ISIS facility, STFC Rutherford Appleton Laboratory, Chilton, Didcot, Oxfordshire, OX11 0QX, United Kingdom \\ 3 Clarendon Laboratory, Department of Physics, University of Oxford, Parks Road, Oxford OX1 3PU, United Kingdom
}

\begin{abstract}
Spin-lattice coupling and ferroelectric polarization associated with the magnetic ordering in a geometrically frustrated delafossite $\mathrm{AgFeO}_{2}$ have been studied by high resolution neutron powder diffraction and dielectric measurements. The cycloidal magnetic ground state found in this material and the character of the lattice distortions are unprecedented in the family of the delafossite compounds, implying a peculiar mechanism lifting the geometrical frustration.
\end{abstract}

\section{Introduction}

In recent years, physics of geometric frustration in triangular lattice antiferromagnets (TLA) has renewed a great interest due to correlation between frustrated magnetism and structural degrees of freedom as found in a number of multiferroic materials.[1,2] $A B \mathrm{O}_{2}$ compounds with delafossite or $\alpha-\mathrm{NaFeO}_{2}$ type of the crystal structure, where $A$-site is nonmagnetic $(\mathrm{Ag}, \mathrm{Cu}, \mathrm{Pr}, \mathrm{Pd}$, or $\mathrm{Na})$ and $B$-site is magnetic $(\mathrm{Cr}, \mathrm{Fe}$ or $\mathrm{Ni})$, [3] have provided a great opportunity to study the frustrated magnetism in TLA. Especially, the cases of $\mathrm{CuFeO}_{2}$ (pure and doped with $\mathrm{Al}$ or $\mathrm{Ga}$ ) and $\mathrm{CuCrO}_{2}$ have been intensively studied in last several years due to the spin-driven improper ferroelectricity and spinlattice coupling phenomena.[4-10]

$\mathrm{AgFeO}$ 2 belongs to the delafossite $A B \mathrm{O}_{2}$ family of compounds with rhombohedral $R \overline{3} m$ symmetry. Its crystal structure, with the unit cell parameters $a=b=3.0391(1) \AA$ and $c=18.5899(9) \AA$ at room temperature, is shown in Fig. 1. Although the other delafossites, such as $\mathrm{CuFeO}_{2}$ and $\mathrm{CuCrO}_{2}$, have been extensively explored as frustrated and multiferroic magnets, [4-10] $\mathrm{AgFeO}_{2}$ has not been studied very well, due to the lack of high quality samples. Although one paper has reported the neutron diffraction experiments recently, the magnetic structure was not determined at that time.[11] Very recently, Tsujimoto et al. have succeeded to synthesize $\mathrm{AgFeO}_{2}$ under high pressure.[12] Using these samples, we performed neutron diffraction and dielectric measurements which revealed two magnetic phase transitions at $T_{\mathrm{N} 1}=15 \mathrm{~K}$ and $T_{\mathrm{N} 2}=9 \mathrm{~K}$.[13] In the temperature range $T_{\mathrm{N} 2} \leq T \leq T_{\mathrm{N} 1}$ (ICM1 phase), the magnetic structure is a spin-density wave with a temperature dependent incommensurate modulation $\mathbf{k}=\left(\overline{1} q \frac{1}{2}\right)$. Below $T_{\mathrm{N} 2}$ (ICM2 phase), the magnetic structure turns into elliptical cycloid with the incommensurate propagation vector $\mathbf{k}=\left(\frac{1}{2} q \frac{1}{2}\right)$, giving rise to electric polarization in accordance with the inverse Dzyaloshinskii-Moriya (DM) mechanism.[13]

\footnotetext{
a e-mail: terada.noriki@nims.go.jp
}

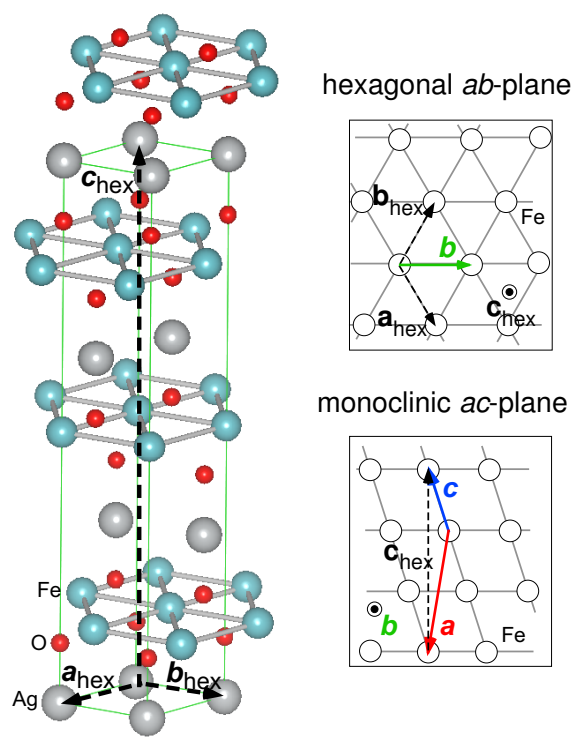

Fig. 1. (left) Crystal structure of delafossite $\mathrm{AgFeO}_{2}$ with hexagonal unit cell. (right) Relationship between hexagonal and monoclinic bases.

In this paper, we review the magnetic ordering and ferroelectricity as well as we provide the results of high-resolution neutron diffraction experiments revealing the strong magnetoelastic coupling in the frustrated spin system of $\mathrm{AgFeO}_{2}$.

\section{Experimental details}

The powder sample of $\mathrm{AgFeO}_{2}$ was prepared under high pressure as described in Ref.[12] High resolution neutron diffraction measurements were carried out at the ISIS facility (UK) using two time-of-flight diffractometers, HRPD [14] to determine the structural parameters, and WISH [15] to analyse the magnetic ordering. 


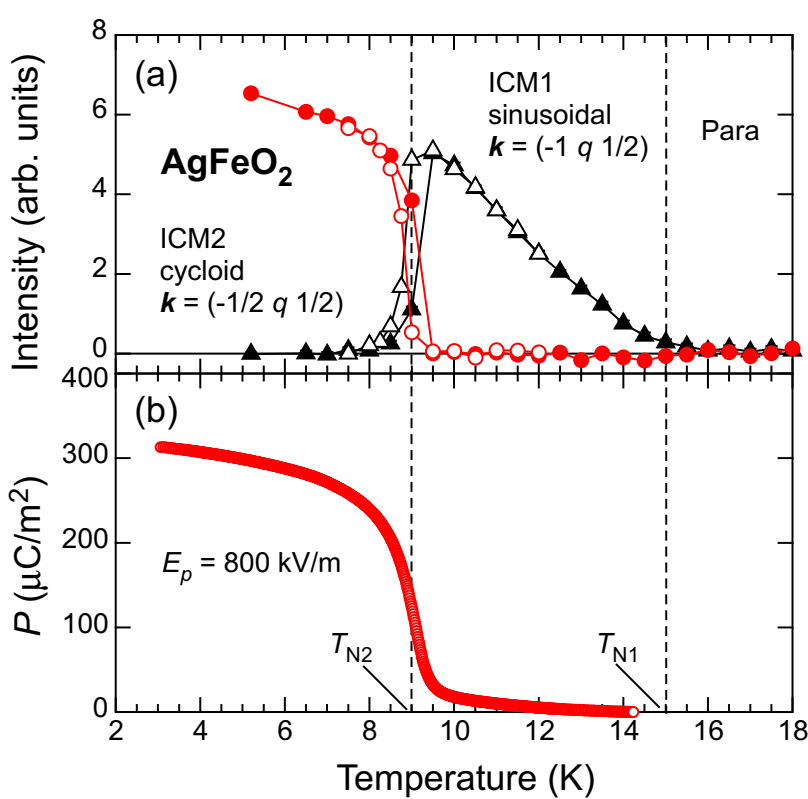

Fig. 2. Temperature dependence of (a) magnetic neutron intensity measured on WISH diffractometer and (b) ferroelectric polarization in $\mathrm{AgFeO}_{2}$. Closed and open symbols denote data on heating and cooling, respectively. Dotted lines show the magnetic phase transition temperatures.

The ferroelectric polarization was determined by pyroelectric current measurements with the poling electric field being $800 \mathrm{kV} / \mathrm{m}$.

\section{Results and Discussion}

\subsection{Magnetic orderings and ferroelectricity}

Let us first briefly describe the spin ordering in $\mathrm{AgFeO}_{2}$ accompanied by structural distortions reducing the rhombohedral symmetry of the paramagnetic phase down to monoclinic. The magnetic reflections indexed by the propagation vector $\mathbf{k}=\left(\overline{1} q \frac{1}{2}\right)$ refereeing to the monoclinic cell shown in Fig.1 (right) have been observed below $T_{\mathrm{N} 1}=15$ $\mathrm{K}$ and successfully modeled in the collinear sinusoidally modulated spin structure. The incommensurate modulation depends on temperature and in the range of stability of the ICM1 phase $9 \mathrm{~K} \leq T \leq 15 \mathrm{~K}$ varies as $0.383 \leq$ $q \leq 0.389$. The deduced centrosymmetric magnetic point group, $2 / m 1^{\prime}$, is consistent with the observed monoclinic peak splitting (discussed in the next section) and the dielectric measurements testifying the lack of polarization just below the first transition. Both k-vector and magnetic structure are almost the same as in the intermediate temperature phase of $\mathrm{CuFeO}_{2}$.[16,17] The spin direction, however, is significantly different in these compounds, suggesting that the $A$-site cations play important role determining the magnetic anisotropy of the $\mathrm{Fe}^{3+}$ spins.

Below the second transition at $T_{\mathrm{N} 2}=9 \mathrm{~K}$, a cycloidal magnetic structure with propagation vector $\mathbf{k}=\left(\frac{1}{2} q \simeq\right.$ $0.205 \frac{1}{2}$ ) and elliptical modulation is stabilized. One of the cycloidal axis coincides with the monoclinic $b$ axis and another is almost parallel to the hexagonal $c$ axis.[13] Note that the cycloidal magnetic ground state in $\mathrm{AgFeO}_{2}$ is completely different from the ground states in other members

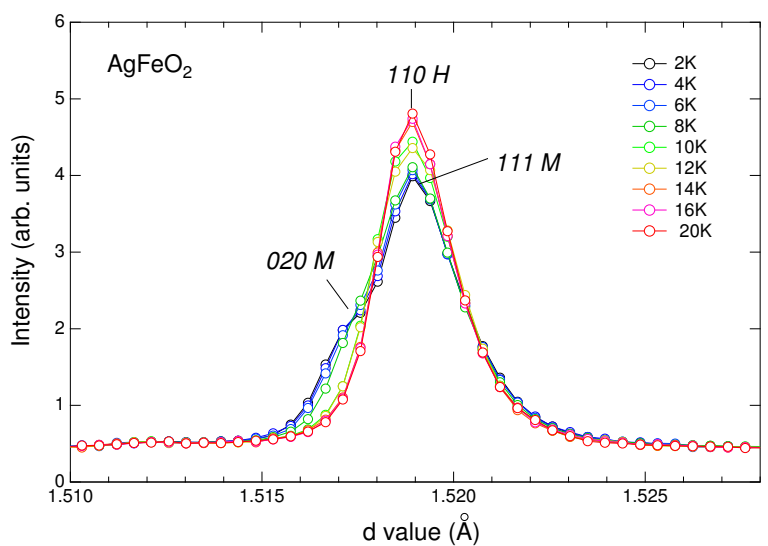

Fig. 3. Temperature dependence of the hexagonal 110 nuclear diffraction profile of $\mathrm{AgFeO}_{2}$. The data were measured on HRPD at increasing temperature. Below $T_{\mathrm{N} 1}=15 \mathrm{~K}$, the peak is split into two components indexed as 020 and 111 in the monoclinic cell.

of the $\mathrm{ABO}_{2}$ family, in particular, from the collinear ordering in $\mathrm{CuFeO}_{2}$ and proper screw state in both $\mathrm{CuCrO}_{2}$ and $\mathrm{CuFe}_{1-x} \mathrm{Ga}_{x} \mathrm{O}_{2}$.[8,10,18] This implies that the exchange interactions among the spins on the $B$ sites as well as their magnetic anisotropy are significantly affected by the substitution of the nonmagnetic $A$-site cations. The importance of $\mathrm{Cu}$ ions in $\mathrm{CuFeO}_{2}$ has been pointed out by Malvestuto et al. [19] in the recent X-ray absorption spectroscopy measurements.

As shown in Fig. 2(b), ferroelectric polarization $\mathbf{P}$ appears below $T_{\mathrm{N} 2}$, which is consistent with the polar magnetic point group, $m 1^{\prime}$, of the cycloidal ICM2 phase. The temperature dependence of $\mathbf{P}$ clearly follows the magnetic Bragg intensity in the ICM2 phase (Fig. 2(a)). The powder averaged value of $P \sim 300 \mu \mathrm{C} / \mathrm{m}^{2}$ corresponds to the

Table 1. Results of the Rietveld refinement of the neutron powder diffraction data collected on HRPD. At $\mathrm{T}=20 \mathrm{~K}$, the symmetry is $R \overline{3} m$ with $\mathrm{Ag}, \mathrm{Fe}$ and $\mathrm{O}$ at the Wyckoff positions, $3 a(0,0,0)$, $3 b(0,0,1 / 2)$ and $6 c(0,0, z)$, respectively. At both $T=10 \mathrm{~K}$ and $T=4 \mathrm{~K}$, the structure was refined in $C 2 / m$ space group with the corresponding occupied positions, $2 d(0,1 / 2,1 / 2)$ for $\mathrm{Ag}, 2 a$ $(0,0,0)$ for $\mathrm{Fe}$, and $4 i(x, 0, z)$ for $\mathrm{O}$.

\begin{tabular}{lcll}
\hline \hline & & & \\
Temperature & $20 \mathrm{~K}$ & $10 \mathrm{~K}$ & \multicolumn{1}{c}{$4 \mathrm{~K}$} \\
& & & \\
\hline & & & \\
Space group & $R \overline{3} m$ & \multicolumn{1}{c}{$C 2 / m$} & $C 2 / m(C m)$ \\
Cell parameters & & & \\
$a(\AA)$ & $3.03273(1)$ & $12.51261(10)$ & $12.51578(11)$ \\
$b(\AA)$ & - & $3.03234(7)$ & $3.02990(2)$ \\
$c(\AA)$ & $18.5838(2)$ & $6.43886(6)$ & $6.44071(6)$ \\
$\beta($ deg $)$ & - & $156.1722(6)$ & $156.1732(3)$ \\
Atomic positions & & & \\
$\mathrm{O} x$ & - & $0.3886(11)$ & $0.3900(6)$ \\
$\mathrm{O} z$ & $0.11194(8)$ & $0.6128(23)$ & $0.6154(11)$ \\
$R_{\text {Bragg }}(\%)$ & 5.45 & 5.57 & 4.56 \\
$\chi^{2}$ & 4.23 & 4.63 & 4.15 \\
& & & \\
\hline \hline
\end{tabular}



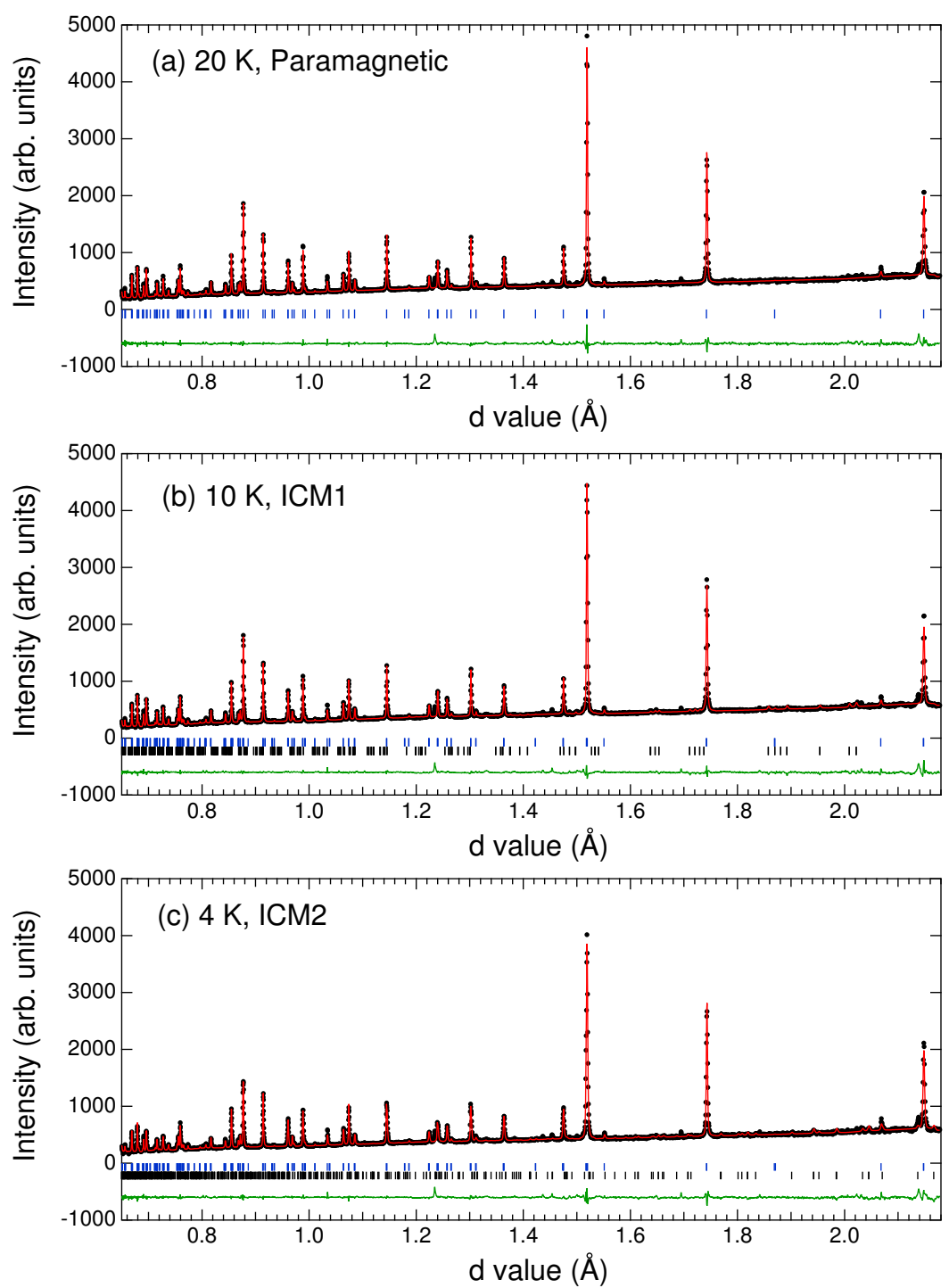

Fig. 4. Typical results of the Rietveld refinement of the experimental data collected on HRPD at (a) $20 \mathrm{~K}$, (b) $10 \mathrm{~K}$ and (c) $4 \mathrm{~K}$. The refinements were performed with FullProf program.[20] The vertical bars indicate nuclear (upper row) and magnetic (bottom row) Bragg peaks. The refined parameters and reliability factors are listed in Table 1.

intrinsic value of $600 \mu \mathrm{C} / \mathrm{m}^{2}$ in a single crystal, which is comparable with the improper polarization in other multiferroics with noncollinear spin ordering.[4] As discussed in our previous paper,[13] the ferroelectric polarization can be understood in terms of the inverse Dzyloshinskii-Moriya mechanism.

\subsection{Lattice distortion}

At high temperature in the paramagnetic phase, the crystal structure of the $\mathrm{AgFeO}_{2}$ is well described by the rhombohedral $R \overline{3} m$ symmetry. The structural parameters refined at $T=20 \mathrm{~K}$ are summarized in Table 1 and the quality of the fit is demonstrated in Fig. 4(a). The situation, however, changes below the magnetic phase transition at $T_{\mathrm{N} 1}=15$ $K$. The spin ordering results in a clear splitting some of the nuclear peaks as shown in Fig. 3, where the temperature evolution of the (110) reflection is presented. While this reflection is a single peak above $T_{\mathrm{N} 1}$, it separates into two components below this temperature. The splitting progressively increases with decreasing temperature in correlation with the development of the magnetic intensity and the associated order parameter.

In accordance with the $2 / m 1^{\prime}$ symmetry of the spin ordering in the ICM1 phase, which allows the coupling between the magnetic order parameter and the corresponding symmetarized combinations of the macroscopic strain components reducing the crystal structure symmetry down to monoclinic $C 2 / \mathrm{m}$, the nuclear structure of the low temperature phase has been described using this space group (Table 1). The relation between the hexagonal and monoclinic cells are shown in Fig. 1 (right). It should be pointed out that the incommensurate nature of the magnetic ordering necessarily implies the existence of structural modulations with the propagation vectors $2 n \mathbf{k}_{\text {magnetic }}(n$ is integer). However, the corresponding satellites were not observed in the present powder diffraction experiments and therefore only coupling with macroscopic quantities was taken into account. 
In the ICM2 phase, in addition to the monoclinic strains, a macroscopic polarization is coupled to the lattice distortions due to the polar character of the cycloidal magnetic ordering. Attempts to refine the crystal structure using the polar $\mathrm{Cm}$ space group did not result in any improvement of the fitting quality and therefore the centrosymmetric $C 2 / m$ space group was used over all temperatures in both ICM1 and ICM2 phases (Fig. 4(b),(c)).

In spite of the common low-temperature monoclinic symmetry in both $\mathrm{AgFeO}_{2}$ and $\mathrm{CuFeO}_{2}$ delafossites, the quantitative character of their lattice distortions is essentially different. In particularly, the $b$ axis in the monoclinic setting contracts below the magnetic phase transition in $\mathrm{AgFeO}_{2}$ and elongates in $\mathrm{CuFeO}_{2}$, implying different mechanisms of releasing the spin frustration in these compounds.

\section{Summary}

We have studied the magnetic ordering, ferroelectricity, and crystal lattice distortions in the multiferroic delafossite $\mathrm{AgFeO}_{2}$. Two magnetic phase transitions at $T_{\mathrm{N} 1}=15$ $\mathrm{K}$ and $T_{\mathrm{N} 2}=9 \mathrm{~K}$ have been revealed and the corresponding magnetic and crystal structures were successfully refined. In the temperature range $9 \mathrm{~K} \leq T \leq 15 \mathrm{~K}$, the magnetic structure is a non-polar $\left(2 / m 1^{\prime}\right)$ spin density wave with the incommensurate propagation vector $\mathbf{k}=\left(\overline{1} q \frac{1}{2}\right)$ being dependent on temperature. Below $9 \mathrm{~K}$, the magnetic structure turns into the polar $\left(m 1^{\prime}\right)$ elliptical cycloid with $\mathbf{k}=\left(\frac{1}{2} q \frac{1}{2}\right)$, giving rise to the macroscopic polarization. Analysis of the high resolution neutron diffraction data revealed a clear lattice distortions associated with the onset of the magnetic ordering. The deduced monoclinic symmetry is consistent with the symmetry of the magnetic order parameter and indicates a strong coupling of the latter with the macroscopic strains. The quantitative analysis of the lattice distortions points to a different mechanism lifting the geometrical frustration in $\mathrm{AgFeO}_{2}$ than in the case of the well studied $\mathrm{CuFeO}_{2}$ delafossite.

\section{Acknowledgement}

The images shown in Fig. 1 were depicted using the software VESTA[21] developed by K. Monma. This work is supported by an international collaboration research program, "Young Researcher Overseas Visits Program for Vitalizing Brain Circulation" of JSPS. N.T. is supported by the JSPS Postdoctoral Fellowships for Research Abroad.
5. S. Seki, Y. Yamasaki, Y. Shiomi, S. Iguchi, Y. Onose and Y. Tokura, Phys. Rev. B 75, (2007) 100403(R).

6. N. Terada, T. Nakajima, S. Mitsuda, H. Kitazawa, K. Kaneko and N. Metoki, Phys. Rev. B 78, (2008) 014101.

7. N. Terada, S. Mitsuda, H. Ohsumi and K. Tajima, J. Phys. Soc. Jpn. 75, (2006) 023602.

8. F. Ye, Y. Ren, Q. Huang, J. A. Fernandez-Baca, P. Dai, J. W. Lynn and T. Kimura, Phys. Rev. B 73, (2006) 220404(R).

9. N. Terada, Y. Narumi, Y. Sawai, K. Katsumata, U. Staub, Y. Tanaka, A. Kikkawa, T. Fukui, K. Kindo, T. Yamamoto, R. Kanmuri, M. Hagiwara, H. Toyokawa, T. Ishikawa, and H. Kitamura, Phys. Rev. B 75, (2007) 224411.

10. S. Seki, Y. Onose and Y. Tokura, Phys. Rev. Lett. 101, (2008) 067204.

11. A. Vasiliev, O. Volkova, I. Presniakov, A. Baranov, G. Demazeau, J-M. Broto, M. Millot, N. Leps. R. Klingeler, B. Büchner, M. B. Stone and A. Zheludev, J. Phys. Condens. Matter 22, (2009) 016007.

12. Y. Tsujimoto et al. unpublished

13. N. Terada, D. D. Khalyavin, P. Manuel, Y. Tsujimoto, K. Knight, P. G. Radaelli, H. S. Suzuki and H. Kitazawa, Phys. Rev. Lett. 109, (2012) 097203.

14. R. M. Ibberson, W. I. F. David, and K. S. Knight, The High- Resolution Powder Diffractometer (HRPD) at ISIS - a User Guide, Report, RAL-92-031 (Rutherford Appleton Laboratory, Chilton, Didcot, England, 1992).

15. L. C. Chapon, P. Manuel, P. G. Radaelli, C. Benson, L. Perrott, S. Ansell, N. J. Rhodes, D. Raspino, D. Duxbury, E. Spill and J. Norris, Neutron News 22, (2011) 22.

16. S. Mitsuda, N. Kasahara, T. Uno and M. Mase, J. Phys. Soc. Jpn. 67, (1998) 4026.

17. N. Terada, T. Kawasaki, S. Mitsuda, H. Kimura and Y. Noda, J. Phys. Soc. Jpn. 74, (2005) 1561.

18. T. Nakajima, S. Mitsuda, K. Takahashi, M. Yamano, K. Masuda, H. Yamazaki K. Prokes, K. Kiefer, S. Gerischer, N. Terada H. Kitazawa, M. Matauda, K. Kakurai, H. Kimura, Y. Noda, M. Soda, M. Matsuura, and K. Hirota Phys. Rev. B 79, (2009) 214423.

19. M. Malvestuto, F. Bondino, E. Magnano, T. T. A. Lummen, P. H. M. van Loosdrecht and F. Parmigiani, Phys. Rev. B 83, (2011) 134422.

20. J. Rodriguez-Carvajal, Physica B, 192, (1993) 55.

21. K. Momma and F. Izumi, J. Appl. Crystallogr. 41, (2008) 653.

\section{References}

1. M. Matsuda, H. Ueda, A. Kikkawa, Y. Tanaka, K. Katsumata, Y. Narumi, T. Inami, Y. Ueda and S.-H. Lee, Nature Phys. 3, (2007) 397.

2. T. Kimura, T. Goto, H. Shintani, K. Ishizaka, T. Arima and Y. Tokura, Nature 426, (2003) 55.

3. R. D. Shannon, D. B. Rogers and C. T. Prewitt, Inorg. Chem., 10, (1971) 713.

4. T. Kimura, J. C. Lashley and A. P. Ramirez, Phys. Rev. B 73, (2006) 220401(R). 